\title{
El rol de las bibliotecas en tiempos de COVID-19: reflexiones y propuestas
}

\section{The role of libraries in the time of Covid-19: reflections and proposals}

\author{
Maria Luisa (Nina) Alonso ${ }^{1}$ \\ Fundación Educar para el Futuro, Colegio Virgen de Europa. Madrid, España \\ dralonsosoroa@gmail.com \\ ORCID 0000-0002-9078-6339
}

\section{Aline Frederico ${ }^{2}$ \\ Pontifícia Universidade Católica de São Paulo / Universidade Federal do Rio de Janeiro afrederico@pucsp.br / alinefrederico@ufrj.br \\ ORCID 0000-0002-5670-8548}

Citar como: Alonso, M. L. y Frederico, A. (2020). El rol de las bibliotecas en tiempos de COVID-19: reflexiones y propuestas. Desde el Sur, 12(1), pp. 241-262.

\section{RESUMEN}

Este trabajo hace una reflexión acerca del rol de las bibliotecas como centro de acceso a la literatura y a la cultura para la infancia y la juventud durante la pandemia de la COVID-19. Empieza con una cartografía de las iniciativas dirigidas a este público promovidas por las bibliotecas

1 Doctora por la Universidad de Cambridge, donde colaboró junto con la doctora Frederico en proyectos sobre literatura y productos culturales para niños y jóvenes, en el Centro académico Homerton-Cambridge International Research Centre for Children's Literature. Ha trabajado también en la Universidad de Cambridge asistiendo proyectos sobre el acceso igualitario a la educación del REAL Centre (Research on Equal Access to Learning) de la Facultad de Educación, en estudios sobre el acceso a la educación y la cultura en África subsahariana. Ha coordinado las actividades de organizaciones de lectura de varios países (Il Était une Fois, Les Mots de Zaza) y realizado reseñas sobre literatura para jóvenes para diferentes instituciones. En la actualidad dirige una biblioteca escolar en España acompañando su transformación en un centro de recursos adaptado a los programas del Bachillerato Internacional (biblioteca digital y física conceptual). Coordina la elaboración colaborativa de materiales creativos y actividades culturales y de promoción de la lectura en todos los niveles curriculares. Es coordinadora de proyectos para la red de escuelas asociadas de la Unesco de su centro educativo. 2 Investigadora en literatura y medios digitales para la infancia, lectura digital y multimodalidad. Actualmente es profesora en la Facultad de Comunicación de la Universidad Federal de Río de Janeiro (UFRJ) e investigadora posdoctoral en el Programa de Literatura y Crítica Literaria en la Pontificia Universidad Católica de São Paulo (PUC-SP). Es doctora por la Universidad de Cambridge, donde colaboró junto con la doctora Alonso en proyectos sobre literatura y productos culturales para niños y jóvenes, en el Centro académico HomertonCambridge International Research Centre for Children's Literature, donde ambas desarroIlaron sus investigaciones doctorales. También tiene una maestría en Literatura Infantil de la Universidad de Columbia Británica, Canadá, y una licenciatura en Edición de la Universidad de São Paulo. 
públicas de la ciudad de São Paulo, Brasil, entre marzo y mayo de 2020. Este análisis revela que las acciones digitales de las bibliotecas se concentraron en difusiones audiovisuales de mediación de lectura y de cuentacuentos, accesibles desde las redes sociales. Entretanto, las bibliotecas no han ejercitado con la misma fuerza su papel de curadoras de contenidos digitales, en un tiempo en que estos pasaron a ser la única opción para muchas familias. Proponemos que las bibliotecas subrayen su papel de curaduría y sugerimos el formato podcast como posibilidad de acción colaborativa de calidad estética y cultural entre bibliotecas y sus comunidades.

\section{PALABRAS CLAVE}

COVID-19, biblioteca digital, infancia, podcast, cultura

\section{ABSTRACT}

This essay reflects on the role of libraries in promoting access to literature and culture for children and young people during the COVID-19 pandemic. We begin with an examination of the initiatives aimed at this section of the public promoted by public libraries in the city of São Paulo, Brazil, between March and May 2020. This analysis reveals that the libraries' digital actions were focused mainly on audiovisual material published on social media, in the form of reading mediation and storytelling. Libraries, it would appear, have not devoted the same effort to their role as curators of digital content, at a time when digital content has become the only option for many families. It is suggested, therefore, that libraries emphasize their curatorial role, and that they explore the podcast format as a possible medium for aesthetically and culturally valuable collaboration between libraries and their communities.

\section{KEYWORDS}

COVID-19; digital library; childhood; podcast; culture 


\section{Introducción ${ }^{3}$}

La pandemia de la COVID-19 ha producido un punto de inflexión en el campo de la educación y de la cultura, el cual finalmente nos puede hacer actuar frente al reto de repensar drásticamente los modelos existentes de enseñanza y, también, como solicitan estos días intelectuales y científicos de todo el mundo, un punto de inflexión que nos incite a trabajar en una «reforma profunda de los objetivos, los valores y las economías» (Binoche y Barrau, 2020, párr. 3). La situación que viven las bibliotecas latinoamericanas durante la pandemia hace más necesario que nunca

pensar y a repensar el rol del bibliotecario y de la biblioteca [...] más allá del espacio de la biblioteca, más allá del contenido de los libros, que debe estar enfocado más que nunca en la comunidad a la cual sirve y debe redoblar sus esfuerzos para pensar cómo brindar sus servicios de lectura, de información, de apoyo al desarrollo económico, emocional, social y cultural de su comunidad (Oyarzún, 2020, párr. 9).

En relación con la infancia, las reformas deben poner en relieve alternativas al consumismo masivo e irreflexivo que obstaculiza el acceso a una dieta sana de entretenimiento lúdico y cultural. Necesitamos repensar cómo y qué educación, entretenimiento y cultura se ofrece a los más jóvenes desde entornos en los que es posible una interacción digital fluida, pero también habilitar soluciones creativas y atractivas para contextos en los que la enseñanza o el acceso a la cultura en línea es difícil o simplemente no es posible (un problema relacionado con el impacto dispar de la epidemia en ricos y pobres (Fisher y Bubola, 2020).

Tras más de un mes pegados a múltiples pantallas y bombardeados por todo tipo de contenidos, somos más que nunca conscientes de la falta de concentración y rendimiento que produce la maraña inconmensurable de fuentes de información y contenidos digitales que compiten por nuestra atención. Los niños y jóvenes nacidos en una generación ya digital, o al menos aquellos con condiciones socioeconómicas para estar conectados, pueden encontrar contenido sobre lo que quieran a través de una miríada de canales digitales, pero sin orientación caen más fácilmente en lo más mediático y necesitan apoyo para aprender a administrar su tiempo y establecer prioridades. Para que el juego, entretenimiento y aprendizaje digital en el hogar resulte enriquecedor, se necesita el apoyo de organizaciones

3 Considerando la actualidad del tema y la necesidad, en este trabajo que dialoga con ideas y propuestas recientes en circulación desde la declaración de la pandemia de COVID-19 (11 de marzo de 2020), recurrimos también a artículos publicados en medios de comunicación tradicional no académica ( $\mathrm{y}$, por lo tanto, sin revisión de pares), a la vez que buscamos voces de académicos y expertos en sus áreas de actuación que puedan contribuir al debate propuesto. 
y profesionales competentes a la hora de filtrar, categorizar y priorizar; agentes de confianza, cercanos, abiertos al diálogo, capaces de ofrecer apoyo fiable y contenidos culturales y didácticos digitales diversos, pertinentes (incluyendo formatos digitales que ayudan a descansar de las pantallas) e idealmente personalizados (Fuller, Lizárraga y Gray, 2015).

Aseguran la Organización de las Naciones Unidas para la Educación, la Ciencia y la Cultura (Unesco) y el Centro Regional para el Fomento del Libro en América Latina y el Caribe (Cerlalc) (Aínsa, 1999) que las bibliotecas, grandes y pequeñas, son instituciones que deberían actuar activamente para ocupar las labores descritas. Las bibliotecas en el siglo XXI deberán «convertirse en el tesoro del conocimiento humano, participar en la innovación del conocimiento y convertirse en un eslabón importante en la cadena de innovación del conocimiento ${ }^{4}$ (Shanhong, 2000, p. 1).

Para reflexionar sobre la dieta de entretenimiento lúdico y cultural que han propuesto las bibliotecas latinoamericanas en tiempos de COVID-19, tomamos como punto de partida una pequeña cartografía de las bibliotecas en una ciudad brasileña, São Paulo. Se trata de ver lo que aquí han estado ofreciendo, sabiendo que, si bien hay disparidades grandes en el funcionamiento de las bibliotecas del continente, puede servir de retrato del momento de la epidemia en un lugar concreto y usarse como lugar desde donde abrir un espacio a la reflexión necesario en estos tiempos inciertos.

\section{Panorama de las acciones de las bibliotecas en la pandemia. Estudio de caso: la ciudad de São Paulo, Brasil}

\section{São Paulo: su red de bibliotecas públicas municipales y situación como} epicentro de COVID-19 en Brasil

La ciudad de São Paulo es la más poblada de Latinoamérica, la cuarta más poblada del mundo y el epicentro de la epidemia de COVID-19 en Brasil, con 55741 casos confirmados y 4116 muertos al 29 de mayo de 2020 (19 h) (Ministério da Saúde, 2020). Por este triste protagonismo en la crisis epidemiológica, São Paulo fue elegida como estudio de caso en este ensayo.

La ciudad empezó a tomar medidas de confinamiento el 20 de marzo de 2020, con el cierre del comercio y de estructuras culturales como museos y bibliotecas. Las escuelas públicas se cerraron tres días después. A partir de entonces y hasta el momento en el que se escribe este texto,

4 Traducción de las autoras para «become a treasure-house of human knowledge, participate in knowledge innovation, and become an important link in the knowledge innovation chain». 
siguen cerradas las bibliotecas públicas de la ciudad, sin previsión de reapertura. Las escuelas públicas de la provincia de São Paulo ofrecen clases a distancia mediante una aplicación desarrollada por el gobierno y gracias a un acuerdo con las empresas proveedoras de internet, que permite el acceso a la plataforma por internet móvil sin consumir los datos. Las escuelas municipales desarrollaron un manual escolar que los estudiantes reciben en el domicilio para que lo estudien y lo complementan con contenido accesible en línea mediante la herramienta Google Classroom. Las escuelas privadas, en su gran mayoría, pasaron casi inmediatamente a la educación en línea y utilizaron, además del Google Classroom, otras herramientas como Microsoft Teams, Zoom, Google Meet, Cisco Webex y Moodle.

São Paulo también se destaca por su significativa red municipal de bibliotecas, la más grande del país, con cinco bibliotecas centrales, 51 bibliotecas públicas de barrio, 46 bibliotecas en los centros educacionales unificados (CEU), también en los barrios, y cuatro bibliotecas adscritas a otros centros de cultura de la ciudad. Además, existen servicios de extensión bibliotecaria para atender a las regiones de la ciudad que no tienen bibliotecas físicas.

Las bibliotecas de barrio tuvieron, en 2019, según datos de la Secretaría Municipal de Cultura (2020), una frecuencia de 1366033 visitantes. En 2019 se produjeron 659649 prestamos de un acervo de 1685092 ítems, la gran mayoría libros impresos. Las bibliotecas, bastante activas en la realización de eventos culturales y lúdicos, realizaron 15399 eventos ese mismo año. Estos eventos comprenden actividades relacionadas con el libro y la lectura (como cuentacuentos, mediación de lectura, veladas o charlas con escritores) y también espectáculos, exposiciones, cine, cursos, talleres, etc.

Las acciones de estas bibliotecas se centran tradicionalmente en el contacto presencial con las comunidades. No cuentan con servicios de préstamo digital, aunque el catálogo global puede ser consultado en línea. Los «servicios» digitales se limitan a dar información sobre cada biblioteca: cómo llegar, el histórico de la biblioteca, los servicios disponibles y la programación de eventos. Hay una página con sugerencias de lectura actualizada mensualmente y una página centralizada con la programación de eventos de todo el Sistema Municipal de Bibliotecas (SMB), que también dispone de Facebook, Instagram, un blog y una cuenta en Twitter. Las páginas web de cada biblioteca municipal son extensiones de la página principal del SMB, si bien cada biblioteca tiene una presencia individualizada en las redes sociales, especialmente Facebook (todas las bibliotecas municipales tienen su Facebook), Instagram (la mayoría de las bibliotecas) y YouTube (algunas). 


\section{Acciones de las bibliotecas públicas municipales de São Paulo en tiem-}

\section{pos de COVID-19}

Para recoger una muestra de acciones de las bibliotecas públicas en São Paulo en tiempos de COVID-19 analizamos los canales de comunicación digital del SMB (página web, Facebook e Instagram) y de dos bibliotecas de este sistema: la Biblioteca Infantil y Juvenil Monteiro Lobato (web, Facebook, Instagram y YouTube) y de la Biblioteca de Barrio Brito Broca (web, Facebook e Instagram). Estudiamos las entradas publicadas entre el 13 de marzo y el 20 de mayo de 2020.

La Biblioteca Monteiro Lobato, localizada en la zona central de la ciudad, es la biblioteca infanto-juvenil más antigua del país y un centro de referencia en literatura infantil y juvenil. En 2019, la biblioteca fue la más frecuentada de toda la red municipal, con un total de 91232 visitantes anuales (283 visitantes diarios en promedio) (Secretaria Municipal de Cultura, 2020). Ese año, la biblioteca realizó 884 eventos que contaron con una asistencia de 33959 personas, en su mayoría niños (la biblioteca está especializada en este público). De estos eventos, 300 fueron de artes escénicas, pues la biblioteca cuenta con un teatro. La Biblioteca Monteiro Lobato recibe visitas de escuelas de toda la ciudad, así que su público no se localiza, necesariamente, en la comunidad local del barrio donde se ubica.

La Biblioteca Brito Broca se sitúa en el barrio de Pirituba, en la periferia de São Paulo, aproximadamente a $18 \mathrm{~km}$ del centro. Aunque esté entre las bibliotecas más visitadas de la ciudad, su frecuencia es de menos de la mitad que la de la biblioteca Monteiro Lobato, con 39474 visitantes anuales en 2019 (122 visitantes diarios en promedio) (Secretaria Municipal de Cultura, 2020). También tuvo una programación muy activa, con 795 eventos, la mayoría de ellos encuentros (163) y actuaciones en espacios públicos ${ }^{5}$ (272). La biblioteca está bastante conectada con su comunidad local, un gran barrio mixto en que se mezclan poblaciones mejor y peor abastecidas. Gracias a su localización, la Biblioteca Brito Broca busca dar voz a poblaciones más periféricas y a la cultura local.

Con el cierre de las bibliotecas, la comunicación con las comunidades ha pasado a ser exclusivamente digital, especialmente a través de las redes sociales. El análisis de las entradas en las páginas de Facebook de las bibliotecas revela que hubo un momento inicial de semiparalización en el que estos canales fueron usados principalmente para informar del cierre de las actividades, difundir información oficial sobre la pandemia e indicar

5 En português, son llamadas ocupações (ocupaciones), pues son eventos en los que un grupo ocupa un espacio público de la ciudad para hacer manifestaciones literarias, identitarias, etc. 
a los usuarios dónde buscar información sobre la COVID-19 en fuentes oficiales. Con el paso del tiempo, las bibliotecas pasaron a realizar parte de su programación en línea y a desarrollar nuevas maneras de actuar.

La primera entrada en las redes sociales del SMB sobre una acción de servicio digital para los tiempos de pandemia se publicó el 26 de marzo, con el video del cuentacuentos de la historia de tradición oral João e o remédio pra memória (26 de marzo de 2020) por la Compañía Eureka. Este video marca el principio de la serie Me conte uma história (Cuéntame una historia). El 25 de abril, 35 días después del cierre de las bibliotecas, la Secretaría Municipal de Cultura (2020) lanzó una convocatoria para lo que Ilamó «Bibliotecas Online 2020». La convocatoria invitaba a presentar proyectos digitales para realizarse en colaboración con el SMB siguiendo cuatro líneas de actuación: presentaciones artísticas; vivencias; intervenciones artísticas; y libro, lectura y literatura.

A partir de entonces, la página central de programación de eventos de la página web de la ciudad de São Paulo pasó a contener exclusivamente eventos en línea, en su mayoría presentados en vivo. Se trata de eventos como veladas, cuentacuentos, debates e intervenciones literarias, presentaciones de circo y de música, etc.

La programación infanto-juvenil de las bibliotecas analizadas puede ser categorizada en tres tipos:

1. Mediación de lectura con libros

2. Cuentacuentos y otras presentaciones teatrales de historias

3. Indicaciones de contenidos culturales para los niños y jóvenes

En la primera categoría, destacamos el proyecto «Viajando na leitura!» («iViajando en la lectura!»), una iniciativa individual de la Biblioteca Brito Broca, puesta en marcha ya desde la primera semana de confinamiento. Son lecturas en vivo dos veces por día. La primera es por las mañanas y se dirige al público infantil (desde la primera infancia) dedicada a la lectura de libros álbum. En las tardes se leen diariamente capítulos de la novela Anne de Green Gables (Ana de las Tejas Verdes), de Lucy Maud Montgomery (2019), dirigida a lectores de cualquier edad. Los videos quedan disponibles permanentemente en Facebook y temporalmente en el Instagram de la biblioteca.

La Biblioteca Monteiro Lobato empezó el 16 de mayo con actividades de mediación en línea, con la transmisión semanal de cuentos para bebés (previamente grabados), que luego quedaban disponibles de forma permanente en las redes sociales de la biblioteca. El primer video de la serie trajo una propuesta nueva, pues hace la mediación del libro digital Tem 
bicho que sabe..., de Toni e Laíse (2013), presentado por la bibliotecaria en una pantalla de mesa. El libro fue puesto a disponibilidad gratuitamente por la editorial en formato digital, y luego se convirtió en una recomendación de lectura para los usuarios de la biblioteca.

Los cuentacuentos son la producción más abundante en la página de Facebook del SMB. La serie de videos Me conte uma história comprende 17 episodios cortos con duración de entre un minuto y medio y nueve minutos. Aunque muchos de estos videos se basan en historias publicadas en libros, muestran adaptaciones orales hechas por actores profesionales.

Otras bibliotecas de la red empezaron también iniciativas propias, como la Biblioteca Affonso Taunay (por ejemplo, A casa sonolenta (2020), que produce sus cuentacuentos con montajes en los que personas del equipo de la biblioteca narran extractos de cuentos, cada uno desde su casa. Como parte de la Biblioteca Online 2020, hay también una programación de presentaciones en vivo, de 60 a 90 minutos de duración, en general repetidas varias veces, en fechas distintas, presentadas en las redes sociales de diferentes bibliotecas. Por ejemplo, la presentación de cuentos de la tradición oral Histórias sem nome... até você chegar! (Historias sin nombre... jhasta que usted llegue!), con Sandra Guzmán, fue presentada en vivo 15 veces durante la pandemia, en distintos días y horarios, y a través de canales de Facebook de diferentes bibliotecas. La repetición sorprende, pues era posible compartir la presentación en vivo en las páginas de cada biblioteca simultáneamente y luego dejar disponible la grabación en las redes sociales, para que el público pudiera acceder a ella en cualquier momento. La repetición en vivo es innecesaria en el medio digital, pero puede ser una manera de apoyar a la comunidad artística en tiempos de crisis, pues en otras circunstancias los artistas habrían hecho distintas presentaciones en vivo, con el correspondiente cobro de una tarifa por cada vez.

Las bibliotecas también ofrecen recomendaciones de lectura sobre contenidos digitales y otros productos culturales para la infancia y la juventud. Esta es sin duda la acción que parece, hasta ahora, menos sistemática y quizás menos eficiente de las bibliotecas. La Biblioteca Monteiro Lobato, a pesar de su trayectoria como biblioteca infanto-juvenil de referencia, ha realizado durante la pandemia una tímida labor como recomendadora de artefactos culturales. La atención de la biblioteca en este momento parece estar centrada en las recomendaciones de contenidos que los lectores puedan acceder desde casa, en el confinamiento, y por lo tanto dominan las fuentes digitales. En el análisis realizado de la actividad de la biblioteca en las redes sociales se encontraron 12 entradas de recomendaciones, dos de ellas de libros impresos y las demás de contenidos digitales: de YouTube (4 entradas), blog (1), podcast (1), acceso gratuito 
a e-books (3), y una entrada en la que comparten una selección de libros para adultos organizada por la coordinadora de las bibliotecas. El análisis de estas entradas revela poca iniciativa a la hora de curar contenidos digitales. Por ejemplo, cuando la Biblioteca Monteiro Lobato recomienda la biblioteca digital gratuita del grupo Santillana (1 de abril de 2020), únicamente comparte la entrada de una noticia de periódico, exclusiva para abonados del periódico, sobre dicha biblioteca digital, pero no hay ninguna indicación, por ejemplo, de qué libros podrían ser más interesantes para el público. Es decir, la biblioteca realiza un papel de divulgación y no de curación. Curiosamente, una de las recomendaciones (12 de mayo de 2020) es del blog y canal de YouTube A Cigarra e a Formiga, de la periodista especializada en literatura infanto-juvenil Daisy Carias, que realiza recomendaciones de literatura infantil. También en esta ocasión la biblioteca renuncia a su papel de referencia en la área de literatura infanto-juvenil y hace una transferencia de esta especialidad a otra.

La Biblioteca Brito Broca parece apropiarse de manera más sistemática del rol de curadora de contenido y de esta forma presenta la sección «Nas estantes da biblioteca...» («En las estanterías de la biblioteca...»), con citas de los libros del acervo en imágenes que destaca en sus redes sociales. Los textos de sus entradas en Facebook aportan detalles como la sinopsis y los atractivos de las obras recomendadas. Las recomendaciones se dirigen a jóvenes y adultos, y destacan muchas obras en formato de cómic y novela gráfica (por ejemplo, Calvin e Haroldo de Bill Watterson (7 de abril de 2020). En la sección «No trem da imaginação!» («iEn el tren de la imaginación!») (s. f.), la biblioteca da, una vez a la semana, recomendaciones de libros álbum para los más pequeños. También se realizan recomendaciones dispersas de videos relacionados con temas de inclusión y cultura de la comunidad. Sin embargo resulta poco conveniente el hecho de que los libros recomendados sean impresos y, por tanto, no accesibles en este momento en que la biblioteca está cerrada. Parece una lástima que no exista, en contrapartida, una posición activa de búsqueda y selección de obras disponibles de manera digital.

Desde hace tiempo, el SMB ofrece mensualmente una lista de sugerencias de lectura. En marzo, esta lista de recomendaciones estuvo centrada en lecturas relacionadas con la crisis de la COVID-19, y el tema fueron libros sobre epidemias, pero los libros sugeridos no estaban disponibles de manera digital (25 de marzo de 2020). En abril, las indicaciones fueron de lecturas para quedarse en casa, y esta vez 5 de las 13 indicaciones fueron de dominio público y disponibles en PDF a través del gobierno por el sitio web dominiopublico.gov.br (4 de abril de 2020). Ya en mayo, las recomendaciones fueron de plataformas para acceder a libros, películas y cursos 
de acceso en línea gratuito (6 de mayo de 2020). En las plataformas para acceder a libros, no hay, sin embargo, ninguna recomendación dirigida a niños y jóvenes, aunque algunas bibliotecas, individualmente, hayan publicado en sus páginas recomendaciones de este tipo, como los ejemplos discutidos anteriormente. Hay también muchos otros casos de servicios similares gratuitos para esta audiencia (algunos de forma temporal), pero no se incluyen en estas listas. Es más, las bibliotecas se abstienen de hacer una verdadera curación de contenido ni orientan a los usuarios de las bibliotecas sobre cómo navegar los contenidos o referencias de las páginas web recomendadas. Solo se limitan a mencionar los sitios web, a pesar de que comprenden arduas listas que incluyen centenares o millares de obras cada una. Por otra parte, es relevante que las bibliotecas estén centradas en las formas de acceso digital y los formatos digitales más básicos: libros en PDF. No se ven, por ejemplo, recomendaciones de historias interactivas (como aplicaciones literarias), sino apenas una recomendación de plataforma de audiolibros (lista SMB del 6 de mayo de 2020) y una suelta sobre un podcast (entrada de Facebook de la Biblioteca Monteiro Lobato del 29 de abril de 2020).

Finalmente, un elemento que claramente está ausente hasta ahora en las acciones de las bibliotecas analizadas es la participación de los usuarios de las bibliotecas, especialmente la participación de niños y jóvenes, y la coconstrucción de una programación en tiempos de pandemia. Una iniciativa digna de mención viene de la Biblioteca Brito Broca, con la propuesta «Venha ler ao pôr do sol» («Venga a leer a la puesta del sol»). Debido a que hay una hermosa vista de la puesta del sol desde la biblioteca, esta invita a que sus lectores envíen fotos de sus momentos de lectura durante la puesta del sol, desde sus casas, empezando una serie semanal de entradas. Otra acción, en este caso del SMB, se llama «Diz aí leitor(a)» («Dímelo, lector[a]»), destinada a que los usuarios de la biblioteca compartan recomendaciones de lectura. La serie, sin embargo, parece haberse creado antes de la pandemia y no haber tenido continuidad, dado que hay un único video (del 17 de marzo de 2020) publicado antes del cierre de las bibliotecas.

Acerca de los lenguajes utilizados para la comunicación por las bibliotecas estudiadas, el formato de video se destaca significativamente. En el periodo analizado, en la página de Facebook del SMB de publicaron 64 posts y la mayor parte (36) son en formato de video. Todos los videos publicados son iniciativas de la propia red de bibliotecas. Es notable que no se comparten videos de otras iniciativas de promoción a la lectura que vengan de fuera de la biblioteca. Ya la Biblioteca Brito Broca ha publicado 75 videos en el periodo analizado, la mayoría producidos en vivo y 
luego puestos a disposición permanentemente en su canal de Facebook. La Biblioteca Monteiro Lobato es la única que no ha publicado videos de manera significativa, pero, como se ha mencionado, su producción digital también fue escasa en el periodo y muchos videos fueron presentados en vivo y no quedaron disponibles a posteriori.

\section{Reflexiones acerca del rol de las bibliotecas en la pandemia}

En este momento en que aún vivimos una pandemia, las bibliotecas de São Paulo cada día siguen empezando nuevos canales y nuevas propuestas de actuación, con muestras de flexibilidad y dinamismo, y en busca de nuevos formatos y maneras de actuar. Esta situación parece coincidir con la descripción hecha recientemente por Gonzalo Oyarzún (responsable del Sistema Nacional de Bibliotecas Públicas de Chile), quien, al dar un repaso sobre cómo «la situación que afecta al mundo entero con la pandemia ha impactado también gravemente al mundo del libro y la lectura en nuestra región» (2020, párr. 1), destaca el protagonismo de algunas bibliotecas digitales (como la chilena o la peruana).

En la línea de este ensayo, Oyarzún observa que «esta catástrofe mundial nos obliga a pensar y a repensar el rol del bibliotecario y de la biblioteca, que, más allá del espacio de la biblioteca, más allá del contenido de los libros, debe estar enfocado más que nunca en la comunidad a la cual sirve y debe redoblar sus esfuerzos para pensar cómo brindar sus servicios de lectura, de información, de apoyo al desarrollo económico, emocional, social y cultural de su comunidad» (2020, párr. 9).

Los contenidos digitales educativos, culturales y de entretenimiento a los que puede acceder el público infanto-juvenil se distribuyen en múltiples formatos. Si bien el préstamo bibliotecario digital de e-books y audiolibros por parte de la infancia y la juventud parece residual en todo el continente latinoamericano, existe gran diversidad de opciones en formatos de video, aplicaciones y podcast-radio, todos ellos disponibles desde celulares y otros dispositivos. Los componentes interactivos y multimedia ayudan a crear dinamismo en los entornos de aprendizaje formales e informales. También fomentan la interconectividad y permiten crear o mantener, incluso en tiempos de incertidumbre, comunidades culturales y de aprendizaje interconectadas.

Establecer una presencia en las redes sociales extiende la manifestación física de la biblioteca al espacio virtual y aumenta la visibilidad, el alcance y el impacto de la biblioteca. Sin embargo, más allá de simplemente establecer una presencia social para la biblioteca, un desafío mayor es construir sitios de redes sociales eficaces y atractivos que tengan éxito en adaptar la visibilidad, la voz y la presencia de 
una biblioteca a los contextos, audiencias y culturas únicas dentro de diversos sitios de redes sociales ${ }^{6}$ (Mon, 2015, p. x).

Recogiendo esta idea de la especialista en ciencias de la información Lorri Mon, proponemos que las bibliotecas refuercen su papel de curaduría digital, como especialistas en el acceso a la producción literaria y cultural en cualquier formato frente a sus comunidades. Invitamos en especial a prestar atención a la diversificación de estrategias de difusión en diálogo con las comunidades locales, teniendo en cuenta las características de acceso de las comunidades a las que sirven. Proponemos agudizar la mirada hacia aquellos contenidos digitales y multimedia que pueden ser de más fácil acceso para bibliotecas, bibliotecarios y comunidades de diversa índole, considerando que no todas las bibliotecas ni los niños y jóvenes tienen acceso fluido a computadoras conectados a internet.

En su manifiesto de la biblioteca digital la Unesco y la Federación Internacional de Asociaciones de Bibliotecarios y Bibliotecas (IFLA) subrayan que «subsanar la brecha digital es un factor clave para alcanzar los Objetivos de Desarrollo del Milenio de las Naciones Unidas. El acceso a los recursos informativos y los medios de comunicación contribuye a la salud y la educación, así como al desarrollo cultural y económico» (2013, párr. 1). Existe un gran debate alrededor de la definición de la brecha digital (Chetty et al., 2018). Sin entrar en este debate, por escaparse del objeto de este trabajo, necesitamos, sin embargo, tener en cuenta aquí las dificultades de acceso de parte importante de las poblaciones latinoamericanas a herramientas y plataformas digitales. En la situación que vivimos existe un peligro real de que las disparidades de acceso entre poblaciones se mantengan y aumenten en algunas regiones. Teniendo en cuenta la «tradición de transmisión cultural a través de las bibliotecas muy afianzada en América Latina» (Ainsa, 1999, p. 31), es necesario reconocer la importante labor que las bibliotecas de diversa índole (Chowdhury, 2002), particularmente en el contexto latinoamericano, pueden jugar en la promoción del acceso de la infancia a una dieta sana de entretenimiento lúdico y cultural, que también contemple recursos y contenidos digitales.

El papel de las bibliotecas en su intento de paliar la brecha digital es tratar de poner a disposición de la infancia y juventud (dialogando, escuchando) recursos digitales de educación, entretenimiento y cultura

6 Traducción de las autoras de «Establishing a social media presence extends the library's physical manifestation into virtual space and increases the library's visibility, reach, and impact. However, beyond simply establishing a social presence for the library, a greater challenge is building effective and engaging social media sites that successfully adapt a library's visibility, voice, and presence to the unique contexts, audiences, and cultures within diverse social media sites». 
categorizados o disponibles (curación de recursos), de forma que permitan un acceso fluido desde entornos en los que es posible una interacción digital ágil y desde donde no. Es precisamente en estos contextos donde resulta crucial apostar por dar credibilidad y visibilidad (formación, apoyo, motivación) a expertos e instituciones especializadas en encontrar lectores para los libros y libros para los lectores, de todo tipo y en todos sus formatos (papel, digital, audio, etc.). Como reconoce Michèle Petit, en América Latina la riqueza es la de muchos bibliotecarios profesionales y voluntarios, que se dedican «a las necesidades del público» (Goldin Halfon, Petit y Arizpe, 2018, p. 140). Daniel Goldin, experto mexicano con una amplia mirada sobre el mundo de los libros, la infancia y las comunidades de lectores, al relatar su experiencia de necesidad de adaptarse a los recursos comentaba algo que refleja una posición con la que la mayoría de los bibliotecarios en Latinoamérica puede empatizar: «entendí que, si alguna tecnología debíamos imitar, debían ser esas tecnologías simples, adaptables a cualquier entorno y que tienen efectos múltiples a un coste muy reducido» (Goldin Halfon, Petit y Arizpe, 2018, p. 142). Las bibliotecas y sus creativos bibliotecarios pueden contribuir a habilitar soluciones digitales imaginativas y atractivas para contextos en los que la enseñanza o el acceso a la cultura en línea es difícil o simplemente no es posible. En Brasil, por ejemplo, el $61 \%$ de los domicilios no tiene computadora y el $28 \%$ no tiene acceso a internet. De los que tienen acceso, el 38\% no tiene banda ancha (datos de 2019 del Núcleo de Informação e Coordenação do Ponto BR, 2020).

Parece en especial oportuno prestar atención a herramientas disponibles, que se constituyen como alternativas a los contenidos de entretenimiento en video tan difundidos y que son más accesibles desde los hogares y las bibliotecas en las que el acceso a internet y computadoras es limitado. La disponibilidad de computadoras con una buena conexión a internet es limitada para gran parte de la población latinoamericana. Existen opciones digitales a las que es oportuno prestar atención, con contenidos disponibles desde teléfonos celulares, que el mediador o el bibliotecario deben barajar al ofrecer una dieta digital variada y accesible a su comunidad.

\section{El podcast: oportunidad de comunicación y creación de comu- nidades lectoras durante la crisis}

Una oportunidad para reflexionar es el contenido de audio (accesible cuando la conexión de internet no es óptima) y, en concreto, el desarrollo de podcasts como herramienta innovadora para el aprendizaje, la comunicación (Guertin, 2010) y el entretenimiento. El podcast, contenido de audio especializado bajo demanda, es capaz de fomentar altos niveles de compromiso personal debido a su capacidad única para borrar la distancia 
entre la persona que entrega el contenido y la audiencia. La accesibilidad y la simplicidad de este medio impulsan el compromiso de su audiencia, mientras que la proximidad e intimidad de la relación entre el hablante y el oyente produce una experiencia diferente a las visuales de los videos en momentos en que nuestros cuerpos y mentes necesitan un descanso de tanto tiempo frente a la pantalla.

Si bien el podcasting se ha impulsado a la primera fila de los canales de comunicación según el Centro de Estudios de Internet y Vida Digital de la Universidad de Navarra, el uso de podcasts en contextos educativos y culturales durante estos tiempos complicados, como indica nuestro estudio de caso de las bibliotecas públicas municipales de São Paulo, parece ser marginal, aunque se aprecia una corriente emergente: los podcasts en español y portugués para la infancia (que cuentan activamente con su audiencia) y, en especial, los podcast de ficción. Creemos que en una región donde la trasmisión oral de historias tiene tradicionalmente un papel muy relevante, existen grandes oportunidades para que los mediadores culturales y bibliotecarios hagan de este medio un canal digital importante. Con esta idea pasamos a comentar algunas iniciativas en podcasts que han estado operativas durante la pandemia, con la idea de que mediadores y bibliotecarios puedan incluirlas en la lista de recursos digitales para recomendar a sus jóvenes públicos y familias.

Unicef en Brasil ha elegido el formato podcast para lanzar durante la pandemia una serie diaria de historias dirigidas a los más jóvenes. Busca «ayudar a niños y familias en este momento de distanciamiento social» (s. f.). Consciente de las limitaciones y carencias de acceso digital de parte de la población, Unicef deja clara su motivación para la elección del formato audio: lo importante es que todos los niños, desde cualquier municipio brasileño, puedan acceder al contenido. La serie de podcasts se llama Deixa que eu conto (Déjame que te cuente) (Unicef, s. f.) y está dirigida a niños de educación infantil y primeros años de educación primaria de todo Brasil. Los capítulos no tienen implicación directa de niños en su diseño y desarrollo, sino que todo es hecho por profesionales adultos. Sigue la forma tradicional de cuentacuentos, en la que un narrador adulto especializado narra una historia y dirige actividades. Pensados con objetivos pedagógicos, los capítulos incluyen historias de temática diversa y se esfuerzan en introducir elementos interactivos, como juegos de llamada y respuesta, y órdenes para que los niños bailen, canten o se muevan al son de una música o juego.

Al estudiar opciones que involucren más a un público infantil y juvenil, sondeamos posibilidades disponibles en Brasil en el momento de la pandemia, y tras reflexionar sobre la poca visibilidad de podcasts para 
este público, nos detenemos en iniciativas que parecen inspiradoras si se busca involucrar directamente al público al que se dirigen. Las siguientes iniciativas dejan ver cómo el público infantil y juvenil puede participar activamente en la concepción, el diseño y el desarrollo de series de podcasts, un componente que parece relevante a la hora de fortalecer comunidades en tiempos difíciles.

La serie de podcast brasileña $E$ se... podcast ( $Y$ si... podcast) (Leite, s. f.) se realiza desde el verano del 2019 y ha seguido en marcha durante los tiempos de la pandemia. En esta serie niños y niñas hacen semanalmente preguntas y dialogan entre ellos y con el coordinador adulto del programa, sobre las cuestiones más diversas desde un punto de vista imaginativo. Se trata de reflexionar (diálogo entre adulto y niños) sobre qué ocurriría si algunas cuestiones de la vida fueran distintas de como son. Así, los niños preguntan en cada capítulo cuestiones como: « $i$ si fueras la última persona de la Tierra?» o « ¿Y si los dragones existieran?». Con el paso del tiempo la serie ha incorporado a adultos expertos en distintas materias que conversan con los niños. Si bien no es una serie de ficción, se trata de historias creativas en las que el interés de los niños, a través de sus preguntas, dirige cada capítulo. La serie está abierta a colaboraciones de nuevos oyentes y tiene una periodicidad interesante para que niños y niñas de distintas comunidades puedan dar seguimiento continuado, y aporten otro elemento más a su dieta lúdica imaginativa y cultural. Puede servir para inspirar a los bibliotecarios para otras nuevas creaciones en podcast o en otros formatos.

En la misma línea de podcasts creados entre adultos y niños conversando destacamos aquí una serie llamada Panela panelinha (Sartén sartencita) (Panela, s. f.) protagonizada por un abuelo y su nieto, en la que, tomando como excusa la realización de recetas culinarias, exploran distintos asuntos interesantes del mundo en el que vivimos, del pasado, de historias reales y ficcionales, etc. Como dice la propia descripción del podcast: «Investigando el origen y la preparación de los alimentos, la pareja viaja en el tiempo y en el espacio para vivir sabrosas aventuras». Por ejemplo, con la excusa de preparar una receta de pescado, abuelo y nieto dialogan de las profundidades del océano, hablan del libro de Julio Verne Veinte mil leguas de viaje submarino e incluso construyen sus propias narrativas sobre los sueños del nieto o las vivencias del abuelo cuando era niño. Se trata de un podcast que lleva produciéndose seis meses.

Fuera de Brasil, pero lógicamente accesibles desde cualquier entorno al tratarse de contenido digital, ponemos la mirada en otras ideas que pueden inspirar a bibliotecarios interesados en impulsar la creación de podcasts o de buscar contenidos en formato de audio para sus comunidades. 
Por su desarrollo colaborativo entre miembros de una comunidad educativa global, destaca la serie de podcasts ANTI Bélicos, un mundo de historias para la paz (Colegio Virgen de Europa, s. f.), realizada desde una biblioteca escolar con la colaboración de escuelas de todo el mundo, principalmente procedentes de la red de escuelas asociadas de la Unesco. Se trata de una iniciativa que recoge textos que hablan del sinsentido de las guerras y sobre la paz agrupados por capítulos, uno por continente, de forma que cada capítulo incluye textos representativos de distintas culturas sobre la paz. Esta serie se ha elaborado uniendo las grabaciones que han hecho principalmente niños y jóvenes, pero también algunos profesores, desde escuelas de los cinco continentes, mediante teléfonos celulares y envío de grabaciones por servicios de mensajería digital a la escuela. Esta coordina, edita y después transmite los capítulos, a través de las plataformas de podcasts y redes sociales. La ventaja de este tipo de iniciativa es que parece fácilmente replicable por otras comunidades y que no necesita disponer de conexiones a internet optimizadas o de dispositivos costosos para poder desarrollarla.

Existen otras ideas interesantes desarrolladas en formato podcast en otros contextos, que pueden servir de inspiración si se adaptan a las necesidades y características de las comunidades a las que distintas bibliotecas sirven, pues se centran en la activa participación del público infantil y juvenil en su concepción. Un ejemplo es el podcast estadounidense Story pirates (Gimlet Media, s. f.), en que niños y niñas participan en la elaboración de las historias que transmiten, en la narración o en entrevistas. Si bien las autoras de este artículo desconocen la existencia de podcasts en español o en portugués que tengan el formato de club de lectura, parece una idea interesante para desarrollar, a partir de las bibliotecas y con la participación del público infantil o juvenil de cada comunidad. En otros idiomas existen podcast-clubs de lectura de gran difusión, en que los que niños o jóvenes recomiendan a gente de su edad libros, videojuegos, aplicaciones e historias en formatos diversos. A menudo, los capítulos incluyen la lectura de algún pasaje. Los más mediáticos y con mayores recursos incluso cuentan con periodistas especializados y actores famosos entrevistando a niños y jóvenes o siendo entrevistados.

\section{Conclusiones}

En este trabajo reflexionamos a partir de un estudio de caso de las bibliotecas públicas municipales de la ciudad de São Paulo, especialmente de los servicios dirigidos al público infanto-juvenil, sobre la actuación y el rol de las bibliotecas durante la pandemia de COVID-19. Vemos que los servicios socioculturales prestados por esta red de bibliotecas antes de la crisis estaban profundamente basados en actividades culturales 
presenciales y en el préstamo de materiales analógicos. Con el cierre de las bibliotecas, hubo un momento inicial de paralización de los servicios para luego retomarse las actividades, sobre todo por medio de las redes sociales. Destacaron en cantidad las actividades relacionadas con la producción de contenido audiovisual propio, especialmente la mediación lectora y los cuentacuentos, con algunas iniciativas realizadas de manera centralizada por la coordinación de la red, y otras iniciativas individuales de cada biblioteca. Así, vemos que el rol de las bibliotecas en la realización de eventos culturales se ha convertido rápidamente al medio digital, mientras que su rol como proveedoras de acceso a la lectura y contenidos editoriales fue interrumpido, pues el préstamo digital no está disponible en esta red.

Aunque el momento y el contexto sean desafiantes, destacamos a lo largo del ensayo algunas posibilidades de actividades y servicios, considerando las limitaciones tecnológicas, pero también valorando la notoria creatividad de los bibliotecarios en nuestro continente latinoamericano, que son capaces no solo de mantener las bibliotecas activas en la promoción de la lectura y la cultura y el acceso a bienes culturales, información y servicios, sino también de revalorizar su rol como articuladores de sus comunidades, aunque sea a distancia. En primer lugar, resaltamos la necesidad de que las bibliotecas asuman su papel de curadoras de contenidos, teniendo en cuenta la gran producción cultural digital existente, que incluye libros y literatura, a la que se puede acceder de manera gratuita. Como conocedoras de las necesidades de sus comunidades, las bibliotecas tienen un importante rol al evaluar, seleccionar e indicar estos contenidos de acuerdo con las necesidades locales, un servicio para el cual necesitan pocos recursos. Además, recomendamos la exploración de formatos alternativos al audiovisual y de fácil producción —y resaltamos el podcast - para articular una producción cultural local que involucre a la comunidad, sobre todo las posibilidades de participación de niños y jóvenes. Presentamos ejemplos de podcasts brasileños e internacionales que involucran esta audiencia directamente, como ejemplos de contenidos culturales gratuitos que pueden enriquecer a las comunidades locales y servir también de inspiración para iniciativas propias de las bibliotecas con sus comunidades.

Nos enfrentamos a un escenario global que a corto y medio plazo incluirá de forma intermitente medidas de distanciamiento social (Kissler et al., 2020) que no permitirán ni el consumo cultural ni la enseñanza cara a cara. ¿Cómo entonces pueden los agentes implicados en la educación y la cultura para la infancia - y entre estos destacamos las bibliotecasadaptarse para facilitar a niños, jóvenes y familias un acceso eficaz e informado a entornos y productos digitales enriquecedores? Debemos 
ver en el momento actual una oportunidad para trabajar en la defensa y fortalecimiento de las bibliotecas, en especial aquellas que juegan un papel importante de cohesión, diálogo e intercambio para las poblaciones menos favorecidas. Es urgente darles apoyo, motivación y herramientas para potenciar las habilidades de los bibliotecarios de curación de contenidos digitales y facilitarles el acceso a tecnologías y aplicaciones básicas.

Las bibliotecas (públicas, escolares, asociativas, comunitarias etc.) se perfilan como aliadas naturales de la transformación hacia lo digital, acelerada por la pandemia, del consumo cultural y de enseñanza. Asegura Anthony Marx, director de una de las mayores bibliotecas del mundo, la Biblioteca Pública de Nueva York, que las tecnologías digitales representan más una oportunidad que una amenaza para las bibliotecas (Wolfe, 2015). Al igual que los libros y los contenidos que migran hacia lo digital (que deja de ser residual para volverse lo mayoritario), lo que aún llamamos biblioteca está abocado a ser un centro de orientación y recursos multimedia.

Jennifer M. Gidley, psicóloga australiana del Instituto para Futuros Sostenibles, asegura que el futuro de una educación y cultura integradas con la tecnología no dependerá de soluciones y proveedores de contenidos (2012), sino de la capacidad de los agentes culturales y educativos de la sociedad de centrarse en las pedagogías y acciones de mediación más que en las herramientas y plataformas digitales. Los bibliotecarios, como agentes culturales y educativos en diálogo con sus comunidades, pueden reflexionar sobre qué estrategias de mediación utilizadas hasta ahora son trasladables a entornos digitales remotos, qué nuevos enfoques e ideas (escalables en función del contexto) podrían incorporarse en distintas comunidades y en cada caso particular en función de las posibilidades y características de cada entorno.

\section{Contribución de autoría}

Maria Luisa (Nina) Alonso y Aline Frederico han participado en la concepción, la recolección de datos, la redacción y la aprobación de la versión final del artículo.

\section{Fuentes de financiamiento}

Esta investigación fue realizada con beca de posdoctorado del Programa Nacional de Pós-Doutorado (PNPD), de la Coordenação de Aperfeiçoamento de Pessoal de Nível Superior (Capes), Brasil, caso número 88882.315383/2019-01.

\section{Conflicto de interés}

Las autoras declaran no tener conflictos de interés. 


\section{REFERENCIAS BIBLIOGRÁFICAS}

Aínsa, F. (1999). Las políticas del libro en América latina. La perspectiva de la Unesco. América. Cahiers Du CRICCAL, 23, 17-36.

Binoche, J. y Barrau, A. (2020). Please, let's not go back to normal. Le Monde. Recuperado de https://www.lemonde.fr/idees/article/2020/05/06/ please-let-s-not-go-back-to-normal_6038793_3232.html

Ministério da Saúde (2020). Painel Coronavírus. Recuperado de https:// covid.saude.gov.br/

Chetty, K., Qigui, L., Gcora, N., Josie, J., Wenwei, L. y Fang, C. (2018). Bridging the digital divide: Measuring digital literacy. Economics: The OpenAccess, Open-Assessment E-Journal, 12(2018-2023), pp. 1-20. Recuperado de https://doi.org/10.5018/economics-ejournal.ja.2018-23

Chowdhury, G. G. (2002). Digital divide: How can digital libraries bridge the gap? En E.-P. Lim, S. Foo, C. Khoo, H. Chen, E. Fox, S. Urs y T. Costantino (Eds.), Digital libraries: People, knowledge, and technology (pp. 379-391). Berlín: Springer Berlin Heidelberg.

Fisher, M. y Bubola, E. (2020). As Coronavirus deepens inequality, inequality worsens its spread. The New York Times. Recuperado de https://www. nytimes.com/2020/03/15/world/europe/coronavirus-inequality.html

Fuller, B., Lizárraga, J. R. y Gray, J. H. (2015). Digital media and latino families: New channels for learning, parenting, and local organizing. Nueva York: The Joan Ganz Cooney Center at Sesame Workshop.

Garner, B. (2019). Teaching students to become digital content curators. Fact or fiction? Cambridge: Cambridge Scholars Publishing.

Gidley, J. M. (2012). Futuros de la educación para una sociedad global en rápido cambio. En F. González (Ed.), Hay futuro. Visiones para un mundo mejor (pp. 411-436). Madrid: BBVA.

Goldin Halfon, D., Petit, M. y Arizpe, E. (2018). Una conversación entre Daniel Goldin Halfon y Michèle Petit, con Evelyn Arizpe como directora de orquesta. Jeunesse: Young People, Texts, Cultures, 10(1), pp. 134-168. Recuperado de https://jeunessejournal.ca/index.php/yptc/article/view/417

Guertin, L. A. (2010). Creating and using podcasts across the disciplines. Currents in Teaching and Learning, 2(2), pp. 4-12

Federación Internacional de Asociaciones de Bibliotecarios y Bibliotecas, IFLA, y Organización de las Naciones Unidas para la Educación, la Ciencia y la Cultura, Unesco (2013). Manifiesto de las IFLA/UNESCO sobre las bibliotecas digitales. Recuperado de https://www.ifla.org/ES/publications/ manifiesto-de-las-ifla-unesco-sobre-las-bibliotecas-digitales 
Kissler, S. M., Tedijanto, C., Goldstein, E., Grad, Y. H. y Lipsitch, M. (2020). Projecting the transmission dynamics of SARS-CoV-2 through the postpandemic period. Science, 368(6493), pp. 860-868. Recuperado de https://doi. org/10.1126/science.abb5793

Mon, L. (2015). Social media and library services. Chapel Hill: Morgan \& Claypool.

Montgomery, L. M. (2019). Anne de Green Gables. Belo Horizonte; São Paulo: Autêntica Infantil e Juvenil.

Núcleo de Informação e Coordenação do Ponto BR (Ed.) (2020). Pesquisa sobre o uso das tecnologias de informação e comunicação nos domicílios brasileiros: TIC domicílios 2019. São Paulo: Comitê Gestor da Internet no Brasil.

Oyarzún, G. (2020). Libros y bibliotecas en tiempos de Zoom. El Comején. Recuperado de https://elcomejen.com/2020/05/16/libros-y-bibliotecasen-tiempos-de-zoom

Secretaria Municipal de Cultura de São Paulo (cidade) (2020). Biblioteca Online 2020. Chamada para interessados em realização de atividades artísticas online. Recuperado de https://www.prefeitura.sp.gov.br/cidade/ secretarias/cultura/bibliotecas/noticias/?p=27784

Secretaria Municipal de Cultura de São Paulo (cidade) (2020). Frequência, matrículas, empréstimos, consultas e acervos dos serviços municipais de leitura vinculados à Coordenação do Sistema Municipal de Bibliotecas, segundo os tipos e serviços municipais de leitura, São Paulo. Recuperado de https://www.prefeitura.sp.gov.br/cidade/upload/consolidacao_2019_ anual_versao_20200330_1588268458.xIsx

Shanhong, T. (2000). Knowledge Management in Libraries in the 21 st Century. Proceedings of the 66th IFLA Council and General Conference. Recuperado de https://archive.ifla.org/IV/ifla66/papers/057-110e.htm

Sistema Municipal de Bibliotecas, SMB (2020). Dicas de Leitura. Fique em Casa. Recuperado de https://www.prefeitura.sp.gov.br/cidade/secretarias/cultura/bibliotecas/noticias/?p=27777

Toni, \& Laíse (2013). Tem bicho que sabe... São Paulo: Bamboozinho.

Wolfe, J. (2015). The 21st Century Library: A conversation with NYPL's Anthony Marx. Forbes. Recuperado de https://www.forbes.com/sites/joshwolfe/2015/06/01/the-21 st-century-library-a-conversation-with-nyplsanthony-marx/\#74fb0ebd3ee5 
DATOS ANALIZADOS: PÁGINAS WEB, REDES SOCIALES Y REDES SOCIALES REFERENCIADAS

Biblioteca Infantojuvenil Monteiro Lobato (s. f.). Entrada de Instagram. Recuperado de https://www.instagram.com/bibliotecaijmonteirolobato/

Biblioteca Infantojuvenil Monteiro Lobato (2020). Gente, olha que bacana! Entrada de Facebook. Recuperado de https://www.facebook.com/bijmlobato/posts/1117855438565821

Biblioteca Infantojuvenil Monteiro Lobato. (2020, 29 de abril). \#Repost @ kiaraterrakikita. Recuperado de https://www.facebook.com/bijmlobato/ posts/1139273926423972

Biblioteca Infantojuvenil Monteiro Lobato (2020, 12 de mayo). Dica da Lobato. Recuperado de https://www.facebook.com/bijmlobato/ posts/1150017092016322

Biblioteca Infantojuvenil Monteiro Lobato. (s. f.). [Entrada de Facebook]. Recuperado de https://www.facebook.com/bijmlobato/

Biblioteca Infantojuvenil Monteiro Lobato (s. f.). [Video de YouTube]. Recuperado de https://www.youtube.com/channel/UCHRN5M5vYfED21yWWQB1Zpw

Biblioteca Pública Affonso Taunay (2020, 1 de mayo). A Casa Sonolenta. Recuperado de https://www.facebook.com/613423105433213/videos/759091844830108

Biblioteca Pública Municipal Brito Broca. (s. f.). [Entrada de Instagram]. Recuperado de https://www.instagram.com/bibliotecabritobroca/

Biblioteca Pública Municipal Brito Broca (2020, 7 de abril). Calvin e Haroldo, de Bill Watterson. Recuperado de https://www.facebook.com/bibliotecabritobroca/posts/3021726817886066

Biblioteca Pública Municipal Brito Broca (s. f.). \#NoTremdalmaginação [hashtag]. Recuperado de https://www.facebook.com/hashtag/ notremdaimagina $\% \mathrm{C} 3 \% \mathrm{~A} 7 \% \mathrm{C} 3 \% \mathrm{~A} 30$

Biblioteca Pública Municipal Brito Broca (s. f.). [Entrada de Facebook]. Recuperado de https://www.facebook.com/bibliotecabritobroca/

Bibliotecas de São Paulo, Sistema Municipal de Bibliotecas, CSMB (s. f.). [Entrada de Instagram]. Recuperado de https://www.instagram.com/smbibliotecas/

BibliotecasSP (2020, marzo 18). DIZ AÍ LEITORA: @kellykellina. Recuperado de https://www.facebook.com/157885720894360/videos/645627302905276

BibliotecasSP (2020, 1 de mayo). João e o Remédio pra Memória. Recuperado de https://www.facebook.com/BibliotecasSP/videos/227927298357958 
BibliotecasSP. (s. f.). [Entrada de Facebbok]. Recuperado de https://www. facebook.com/BibliotecasSP/

BibliotecasSP. (s. f.). [Entrada de Facebook]. Recuperado de https://www. facebook.com/BibliotecasSP/

Sistema Municipal de Bibliotecas, SMB (2020, 4 de abril). Dicas de Leitura - Fique em Casa. Recuperado de https://www.prefeitura.sp.gov.br/cidade/ secretarias/cultura/bibliotecas/noticias/?p=27777

Sistema Municipal de Bibliotecas, SMB (2020, 25 de marzo). Dicas de Leitura - Pandemias. Recuperado de https://www.prefeitura.sp.gov.br/cidade/ secretarias/cultura/bibliotecas/noticias/?p=27732

Sistema Municipal de Bibliotecas, SMB (2020, 6 de mayo). Dicas de Leitura - Livros, filmes e cursos online. Recuperado de https://www.prefeitura. sp.gov.br/cidade/secretarias/cultura/bibliotecas/noticias/?p=27822

Sistema Municipal de Bibliotecas, SMB (s. f.). Biblioteca Infantojuvenil Monteiro Lobato. Recuperado de https://www.prefeitura.sp.gov.br/cidade/secretarias/cultura/bibliotecas/monteiro_lobato/

Sistema Municipal de Bibliotecas, SMB (s.f.). Biblioteca Pública Municipal Brito Broca. Recuperado de https://www.prefeitura.sp.gov.br/cidade/secretarias/cultura/bibliotecas/bibliotecas_bairro/bibliotecas_a_l/britobroca/

Sistema Municipal de Bibliotecas, SMB (s. f.). Bibliotecas. Recuperado de https://www.prefeitura.sp.gov.br/cidade/secretarias/cultura/bibliotecas/

\section{PODCASTS MENCIONADOS}

Colegio Virgen de Europa (Productor) (s. f.). ANTI Bélicos. Recuperado de https://open.spotify.com/

Fondo de las Naciones Unidas para la Infancia, Unicef (Productor) (s. f.). Deixa que eu conto. Recuperado en 30 da Mayo de 2020 de https://open. spotify.com/

Gimlet Media (Productor) (s. f.). Story pirates. Recuperado de https://www. storypirates.com/podcast

Leite, S. (Productor) (s. f.). E se ... podcast. Recuperado de http://www.esepodcast.com.br/

Panela (Productor) (s. f.). Panela Panelinha. Recuperado de https://olapodcasts.com/channels/panelapanelinha 